\title{
Preventive effect of chrologenic acid on permanent hearing loss induced by repeated exposure to noise
}

\author{
Taro Yamaguchi, Naoko Mitsuba, Masanori Yoneyama, Yusuke Onaka, Kiyokazu Ogita \\ Laboratory of Pharmacology, Faculty of Pharmaceutical Sciences, Setsunan University, Japan
}

Most of sensorineural hearing loss is caused by irreversible cochlear injury. Mammalian cochlea is a fragile organ, which is hard to recover once a damage occurs. Therefore, it is important for measures against sensorineural hearing loss to prevent at the onset of the inner ear injury. In the present study, we prepared an animal model of permanent hearing loss, which is caused by repeated exposure to noise at $90-\mathrm{dB}$ SPL, and sought to determine whether chrologenic acid has preventive effect on the hearing loss in this model mouse.

The mice were exposed to an octave-band noise, centered at $8 \mathrm{kHz}(5.4-11.2 \mathrm{kHz})$, at a $90-\mathrm{dB}$ sound pressure level (SPL) for $1 \mathrm{~h}$ per day for 5 days within a sound chamber and measured the auditory threshold at the frequencies of 4, 12, and 20 $\mathrm{kHz}$. Chrologenic acid was orally administered at a dose of 2 or $10 \mathrm{mg} / \mathrm{kg}$ once a day from 3 days before and during noise exposure. Cochleae were dissected into 6 pieces for whole-mount processing of the cochlear epithelium. Synapses were visualized by immunostaining used following primary antibodies: anti-CtBP2 (C-terminal Binding Protein) to quantify pre-synaptic ribbons; anti-GluA2 (Glutamate receptor subunit A2) to quantify post-synaptic receptor patches; antimyosin VIIa to label hair cell cytoplasm.

At the frequencies of 12 and $20 \mathrm{kHz}$, the auditory threshold markedly increased as noise-exposure times-dependent manner. Noise exposure caused the loss of synapses between inner hair cell and cochlear nerve in middle but no significant hair cell loss. Treatment with chrologenic acid at $10 \mathrm{mg} / \mathrm{kg}$ mostly completely prevented the noise-induced elevation of the auditory threshold at 12 and $20 \mathrm{kHz}$ frequencies. Moreover, chrologenic acid protected the loss of synapses after the noise exposure.

Our data suggest that chrologenic acid is a candidate of preventive drugs for sensorineural hearing loss. 